\title{
A CARDINAL NUMBER ASSOCIATED WITH A FAMILY OF SETS ${ }^{1}$
}

\section{SEYMOUR GINSBURG}

Let $U$ be a family of nonempty subsets of an abstract set $R$, partially ordered by set inclusion. ${ }^{2}$ The smallest cardinal number which is the power of the union of a maximal family of incomparable elements of $U$ shall be defined as the "maximal density" of $U$ (md $(U)$ ). The smallest cardinal number which is the md $(V)$ of some coinitial subfamily $V$ of $U$ shall be defined as the "containing maximal density" of $U(\mathrm{cmd}(U))$. The principal result of this paper is Theorem 2, which states that $\prod_{\xi<\alpha}$ cmd $\left(U^{\xi}\right)=\operatorname{cmd}\left(\prod_{\xi<\alpha} U^{\xi}\right)$.

Before turning to our main result we consider the containing maximal density of a ramified family of sets. ${ }^{3}$

THEOREM 1. If $U$ is a ramified family of sets, then the maximal density of $U$ equals the containing maximal density of $U$.

Proof. Let $V=\{E\}$ be a coinitial subfamily of $U=\{D\}$, such that md $(V)=\mathrm{cmd}(U)$. Let $M=\{H\}$ be a maximal family of incomparable elements of $V$ for which md $(V)=p(M) .{ }^{4}$ We shall now show that $M$ is a maximal family of incomparable elements of $U$. Let $D$ be any element in $U$, and $E$ any element of $V$ which is a subset of $D$. The element $E$ certainly exists since $V$ is a coinitial subset of $U$. The family $M$ being a maximal family of incomparable elements of $V$, there exists an element $H$ in $M$ which is comparable with $E$. If $H$ is a subset of $E$, then $H$ is also a subset of $D$. Suppose that $E$ is a subset of $H$. Since $U$ is ramified, it follows that the two elements $D$ and $H$ are comparable. Consequently each element of $U$ is comparable with some element in $M$. Thus $M$ is a maximal family of incomparable elements in $U$. Therefore

$$
\mathrm{md}(U) \leqq \mathrm{md}(V)=\mathrm{cmd}(U) \leqq \mathrm{md}(U) .
$$

Presented to the Society, September 5, 1952; received by the editors November 1 , 1952.

1 This work was supported in part by funds from the Office of Naval Research, Contract N8-ONR-71400 at the University of Michigan.

${ }^{2}$ In the sequel $U$ and $U^{\xi}$ will be a family of subsets of the sets $R$ and $R^{\xi}$ respectively.

${ }^{3}$ The family of sets $U$ is a ramified family of sets if, for each element $E$ in $U$, the family of sets $\{D \mid D \supseteq E, D \in U\}$ is monotone.

4 Let $Y$ be a family of sets. By $p(Y)$ is meant the power of the set which is the set union of the elements in $Y$. If $Y$ consists of only one set, say $A$, then $p(Y)$ is the power of $A$. In this case, $p(A)$ is also used, i.e., $p(A)=p(Y)$. 
Thus $\operatorname{md}(U)=$ cmd $(U)$.

In preparation for Theorem 2, two lemmas are needed.

Lemma 1. The containing maximal density of $U$ is the smallest cardinal number equal to $p(V)$, for some coinitial subfamily $V$ of $U$.

Proof. Let $V=\{E\}$ be any coinitial subfamily of $U$ for which cmd $(U)=$ md $(V)$. Let $M=\left\{E_{v}\right\}$ be a maximal family of incomparable elements of $V$ such that $p(M)=$ md $(V)$. Now the subfamily of $V$,

$$
Y=\left\{E \mid E \subseteq E_{\nu}, E \in V, E_{\nu} \in M, \text { for some } \nu\right\},
$$

is coinitial in $V$. Thus $p(Y) \leqq \mathrm{md}(V)=\mathrm{cmd}(U)$. Now let $p(Z)$ be the smallest cardinal number for some coinitial subfamily $Z$ of $U$. Clearly cmd $(U) \leqq$ md $(Z) \leqq p(Z)$. This completes the proof.

Lemma 2. To each family $U=\{D\}$, there corresponds a coinitial subfamily $V=\{E\}$, and a subfamily, $Y=\left\{D_{\nu} \mid \nu<\delta\right\}$ of $V$, which have the following properties:

(1) each element of $V$ is p-homogeneous ;

(2) $\left\{E \mid E \subseteq D_{\xi}, E \in V\right\} \cap\{E \mid E \subseteq D, E \in V\}=\varnothing$ for $\xi \neq \nu$;

(3) if $\left\{G_{\xi} \mid \xi<\delta\right\}$ is any subfamily of $V$ in which $G_{\xi}$ is a subset of $D_{\xi}$ for each $\xi$, then

$$
p\left(G_{\nu}-\bigcup_{\xi<\nu} D_{\xi}\right)=p\left(D_{\nu}-\bigcup_{\xi<\nu} D_{\xi}\right) \quad(\nu<\delta),
$$

(4) $p\left[U_{v<\delta}\left(D_{\nu}-U_{\xi<\nu} D_{\xi}\right)\right] \geqq \mathrm{cmd}(V)$.

Proof. If $V$ is a coinitial subfamily of $U$ such that md $(V)$ $=\mathrm{cmd}(U)$, and

$$
Z=\{E \mid E \in V, E \text { is } p \text {-homogeneous }\},
$$

then $Z$ is a coinitial subfamily of $U$ for which $\mathrm{md}(Z)=\mathrm{cmd}(U)$. In order to simplify the notation, it is assumed that $U$ has the two properties of $Z$, i.e., (a) md $(U)=\operatorname{cmd}(U)$, and $(b)$ each element $D$ in $U$ is $p$-homogeneous. Well order the elements of $U, D_{0}$ being the first element. Suppose that the family of sets $\left\{D_{\xi} \mid \xi<\lambda\right\}$ has already been defined. Denote by $D_{\lambda}$ the first element $D_{*}$ in $U$ which satisfies the following two conditions:

(c) if $D$ is a subset of $D_{*}$, where $D$ is in $U$, then $D$ is not a subset of $U_{\xi<\lambda} D_{\xi}$;

5 An element $E$ in $V$ is $p$-homogeneous if $p(B)=p(E)$ for each element $B$ in $V$ which is a subset of $E$. See Erdös and Tarski, On families of mutually exclusive sets, Ann. of Math. vol. 44 (1943) pp. 315-329. 
(d) if $D$ is a subset of $D_{*}$, where $D$ is in $U$, then $p\left(D-U_{\xi<\lambda} D_{\xi}\right)$ $=p\left(D_{*}-U_{\xi<\lambda} D_{\xi}\right)$. If the set of elements satisfying (c) is nonempty, then the element $D_{\lambda}$ certainly exists. Let $\left\{D_{\xi} \mid \xi<\delta\right\}$ be a maximal family obtained in this way.

Let $V=\{E\}$ be the subfamily of $U$,

$$
V=\left\{D \mid D \subseteq \bigcup_{\xi<\delta} D_{\xi}, D \in U\right\} .
$$

Conditions (1), (2), (3), and (4) are automatically satisfied. It is necessary to show only that $V$ is a coinitial subfamily of $U$. Suppose that $D_{*}$ is an element of $U$ which contains no element of $V$. Then the element $D_{*}$ satisfies condition (c). This implies that the family $\left\{D_{\xi} \mid \xi<\delta\right\}$ is not maximal. From this contradiction we obtain our conclusion.

We now prove our main result.

Theorem 2. cmd $\left(\prod_{\xi<\alpha} U^{\xi}\right)=\prod_{\xi<\alpha}$ cmd $\left(U^{\xi}\right)$, where by $\prod_{\xi<\alpha} U^{\xi}$ is meant the cartesian product of the $U^{\xi} .6$

Proof. To each family $U^{\xi}$, associate a family of sets, $Y^{\xi}$ $=\left\{D_{\gamma}^{\xi} \mid \gamma<\delta_{\xi}\right\}$, which satisfies the conclusions of Lemma 2. Let $U$ be a coinitial subfamily of $\prod_{\xi} U^{\xi}$ for which $p(U)=\operatorname{md}(U)=\mathrm{cmd}(U)$. In the following, $\beta, \gamma$, and $\xi$ denote indices that range over specified sets of ordinal numbers, whereas $\mu$ and $\nu$ denote indices that range over the class of all $\alpha$-sequences $\sigma=\left\{\sigma_{\xi}\right\}, \xi<\alpha$, where $\sigma_{\xi}<\delta_{\xi}$ for each $\xi$. The notation $\mu<\nu$ refers to the partial ordering defined by

$$
\mu<\nu \text { if and only if } \begin{cases}\mu_{\xi} \leqq \nu_{\xi} & \text { for all } \xi<\alpha, \text { and } \\ \mu_{\xi}<\nu_{\xi} & \text { for some } \xi<\alpha .\end{cases}
$$

For each $\nu, \prod_{\xi} D_{\nu \xi}^{\xi}$ belongs to $\prod_{\xi} U^{\xi}$. Since $U$ is a coinitial subfamily of $\prod_{\xi} U^{\xi}$, there exists an element $D_{v}$ in $U$ such that $D_{\text {v }}$ $\subseteq \prod_{\xi} D_{\nu \xi}^{\xi} . D_{\nu}$ is of the form $D_{\nu}=\prod_{\xi} E_{\nu}^{\xi}$, where for each $\xi<\alpha, E_{v}^{\xi}$ is an element of $U^{\xi}$ and $E_{\nu}^{\xi} \subseteq D_{v_{\xi}}^{\xi}$. It now follows that

$$
\begin{aligned}
\operatorname{cmd}\left(\prod_{\xi} U^{\xi}\right) & =p(U) \geqq p\left(\bigcup_{\nu} D_{\nu}\right) \\
& \geqq p\left[U\left(D_{\nu}-\bigcup_{\mu<\nu} D_{\nu}\right)\right] \geqq p\left[\bigcup_{\nu}\left(D_{\nu}-\bigcup_{\mu<\nu} \prod_{\xi} D_{\mu_{\xi}}^{\xi}\right)\right] .
\end{aligned}
$$

Since each two terms inside the last brackèt are disjoint,

- The cartesian product of the $U^{\xi}$ is the family of sets $\left\{H \mid H=D^{0} \times D^{1} \times \cdots\right.$, $\left.D^{\xi} \in U^{\xi}\right\}$. 


$$
\begin{aligned}
p\left[U \left(D_{\nu}\right.\right. & \left.\left.-\bigcup_{\mu<\nu} \prod_{\xi} D_{\mu_{\xi}}^{\xi}\right)\right] \\
& =\sum_{\nu} p\left(D_{\nu}-\bigcup_{\mu<\nu} \prod_{\xi} D_{\mu_{\xi}}^{\xi}\right) \\
& =\sum_{\nu} p\left(\prod_{\xi} E_{\nu}^{\xi}-\bigcup_{\mu<\nu} \prod_{\xi} D_{\mu_{\xi}}^{\xi}\right),
\end{aligned}
$$

which, since $\Pi_{\xi}\left(E_{\nu}^{\xi}-U_{\beta<\nu_{\xi}} D_{\beta}^{\xi}\right) \subseteq\left(\prod_{\xi} E_{\nu}^{\xi}-U_{\mu<\nu} \Pi_{\xi} D_{\mu \xi}^{\xi}\right)$, is

$$
\begin{aligned}
& \geqq \sum_{\nu} p\left[\prod_{\xi}\left(E_{v}^{\xi}-\bigcup_{\beta<\nu_{\xi}} D_{\beta}^{\xi}\right)\right] \\
& \left.=\sum_{v} \prod_{\xi} p\left(E_{v}^{\xi}-\bigcup_{\beta<\nu_{\xi}} D_{\beta}^{\xi}\right)\right] \\
& =\sum_{\nu} \prod_{\xi} p\left(D_{v_{\xi}}^{\xi}-\bigcup_{\beta<\nu_{\xi}} D_{\beta}^{\xi}\right) \\
& =\prod_{\xi} \sum_{\gamma<\delta_{\xi}} p\left(D_{\gamma}^{\xi}-\bigcup_{\beta<\gamma} D_{\beta}^{\xi}\right) \\
& =\prod_{\xi} p\left[\underset{\gamma<\delta_{\xi}}{U}\left(D_{\gamma}^{\xi}-\underset{\beta<\gamma}{U} D_{\beta}^{\xi}\right)\right] \\
& \geqq \prod_{\xi} \mathrm{cmd}\left(U^{\xi}\right) .
\end{aligned}
$$

Thus cmd $\left(\prod_{\xi} U^{\xi}\right) \geqq \prod_{\xi}$ cmd $\left(U^{\xi}\right)$. Since the reverse inequality is obviously true, it follows that $\prod_{\xi}$ cmd $\left(U^{\xi}\right)=\mathrm{cmd}\left(\prod_{\xi} U^{\xi}\right)$.

For each $\xi<\mu$ let $U^{\xi}$ be a family of infinite subsets of a set $R^{\xi}$. By $\prod_{\xi<\mu}^{\prime} U^{\xi}$ we shall mean the family of sets

$\left\{G \mid G=\prod H^{\xi}\right.$, where $H^{\xi} \in U^{\xi}$, or $H^{\xi}=R^{\xi}$, and all but a finite number of the $H^{\xi}$ are $\left.R^{\xi}\right\}$.

THEOREM 3. cmd $\left(\prod_{\xi}^{\prime} U^{\xi}\right)$ is the smallest cardinal number, call it $\boldsymbol{\aleph}_{\beta}$, which is in the set of cardinal numbers $\left\{\boldsymbol{\aleph}_{\alpha} \mid \boldsymbol{\aleph}_{\alpha}=\mathrm{cmd}\left(U^{\alpha(0)}\right.\right.$ $\left.\left.\times U^{\alpha(1)} \times \cdots \times U^{\alpha(n)}\right) \cdot \prod_{\xi \neq \alpha(j), j \leqq n} p\left(R^{\xi}\right) ; n<\omega\right\}$.

Proof. If $V^{\xi}$ is a coinitial subfamily of $U^{\xi}$, then $\mathrm{cmd}\left(\Pi^{\prime} U^{\xi}\right)$ $=\mathrm{cmd}\left(\Pi^{\prime} V^{\xi}\right)$. Thus, no generality is lost in assuming that for each $\xi$

$$
p\left(U^{\xi}\right)=\mathrm{md}\left(U^{\xi}\right)=\mathrm{cmd}\left(U^{\xi}\right) .
$$

Furthermore, it may be assumed that 


$$
\aleph_{\beta}=\operatorname{cmd}\left(U^{0} \times \cdots \times U^{n}\right) \cdot \prod_{\xi>n} p\left(R^{\xi}\right) .
$$

To prove the theorem, it is sufficient to show that $\aleph_{\beta} \leqq \mathrm{cmd}\left(\Pi^{\prime} U^{\xi}\right)$. Let $U$ be a coinitial subfamily of $\Pi^{\prime} U^{\xi}$ for which $p(U)=$ md $(U)$ $=$ cmd $\left(\Pi^{\prime} U^{\xi}\right)$, and let $D$ be any element of $U$. To simplify the notation, suppose that

$$
D=D^{0} \times \cdots \times D^{n+m} \times \prod_{\xi>n+m} R^{\xi}
$$

Then

$$
\begin{aligned}
\operatorname{cmd}\left(\prod^{\prime} U^{\xi}\right) & =\operatorname{md}(U)=p(U) \geqq p(D) \\
& =p\left(D^{0} \times D^{1} \times \cdots \times D^{n+m}\right) \cdot \prod_{\xi>n+m} p\left(R^{\xi}\right) \\
& \geqq \prod_{\xi>n+m} p\left(R^{\xi}\right) .
\end{aligned}
$$

Also,

$$
\begin{aligned}
\operatorname{cmd}\left(\prod^{\prime} U^{\xi}\right) & \geqq \operatorname{cmd}\left(U^{0} \times \cdots \times U^{n+m}\right) \\
& =\prod_{\xi \leqq n+m} \mathrm{cmd}\left(U^{\xi}\right) \\
& =\prod_{\xi \leqq n+m} p\left(U^{\xi}\right)=p\left(\prod_{\xi \leqq n+m} U^{\xi}\right) .
\end{aligned}
$$

Combining our results we get

$$
\begin{aligned}
\aleph_{\beta} & \leqq p\left(\prod_{j \leqq n+m} U^{j}\right) \cdot p\left(\prod_{\xi>n+m} R^{\xi}\right) \\
& \leqq \mathrm{cmd}\left(\prod_{\xi}^{\prime} U^{\xi}\right) \cdot \mathrm{cmd}\left(\prod_{\xi}^{\prime} U^{\xi}\right) \\
& =\mathrm{cmd}\left(\prod^{\prime} U^{\xi}\right),
\end{aligned}
$$

the last equality resulting from the fact that as each element of $U^{\xi}$ is infinite, $\mathrm{cmd}\left(\Pi^{\prime} U^{\xi}\right)$ is infinite.

UNIVERSITY OF MICHIGAN AND

The University of Miami 